DOI: http://doi.org/10.52716/jprs.v11i2.512

\title{
Decreasing the Tendency of Water to Form Scale and Corrosion in Cooling Towers - South Refineries Company (SRC) - Iraq
}

\author{
Thabit Abbas, Dr.Qays Muthna, Thikra Shihab, Ola Jabur \\ Petroleum Research and Development Center (PRDC) - Ministry of Oil - Iraq \\ Corresponding Author E-mail: thabitabaas@yahoo.com
}

Received 9/2/2020, Accepted 21/9/2020, Published 20/6/2021

This work is licensed under a Creative Commons Attribution 4.0 International License.

\begin{abstract}
$\underline{\text { Abstract }}$
Scale formation and corrosion phenomena are major technical problems at Basra Refinery/ South Refineries Company (SRC). These technical problems are concentrated in cooling towers which are used to supply cooling water to the processing units. The Scales and corrosion products precipitate inside cooling units, heat exchangers and pipeline networks affecting negatively efficiency of refinery's equipment. In this work, a real plant data was collected from four cooling towers which is supplied the coolant to the crude distillation units of the refinery and also from the raw water supplier. The collected data was fitted by Langelier model to predict the tendency of cooling water for scale forming and activation of corrosion. The obtained result shows that the cooling water has a tendency to form scale $\left(\mathrm{CaCO}_{3}\right.$ Precipitation) at cooling tower units. Also the feed water has the same tendency for scale forming.

After analyzing the LSI (Langelier Saturation Index) results, the research team recommends the particular company to overcome this problem by installing a Reverse Osmosis (RO) plant for treating raw water to decrease the concentrations of total dissolved solids (TDS) which result scale and corrosion in the parts of cooling tower as an option to solve the problem.
\end{abstract}

Keywords: $\mathrm{SRC}, \mathrm{CaCO}_{3}$ Precipitation, RO, LSI, TDS, Corrosion. 


\section{Introduction}

Cooling towers are heat exchangers used to dissipate large heat loads to the atmosphere [1]. They are used in a variety of settings including process cooling, power generation cycles, and air-conditioning cycles. All cooling towers used to remove heat from an industrial processor chemical reaction [2]. Proper design of cooling tower system helps in reducing operational and maintenance problems as well as environmental impacts arising from system operation [3].

To control corrosion and scale, maintain the water chemistry of the recirculating water within the parameters listed in Table (1) [4]. The specific measures required vary from system to system and are dependent on the chemistry of the make-up water, the metallurgy of the piping and heat transfer devices exposed to the recirculating water, and all temperatures at which the system will be operating. Bleed/blow-down, the continuous flow of a small portion of the recirculating water to a drain is used to control the concentration of dissolved solids. On rare occasions, this may be adequate to control scale and corrosion. Usually, the chemical scale and corrosion inhibitors are necessary, which raise the allowable level of dissolved solids without the risk of scale and corrosion. Keep the chemically treated water within the guidelines given in Table (1). In cases where bleed/ blow-down alone is being employed for corrosion and scale control without chemical treatment your water treatment specialist may recommend more conservative limits than those shown in Table (2) [5]. 
Table (1) Quality Guidelines for Treated Circulating Water

\begin{tabular}{|c|c|c|c|c|}
\hline \multirow[b]{2}{*}{$\begin{array}{l}\text { Property of } \\
\text { water }\end{array}$} & \multicolumn{4}{|c|}{ Recommended Levels for Various Materials of Construction } \\
\hline & $\begin{array}{l}\text { Galvanized } \\
\text { Steel }\end{array}$ & $\begin{array}{c}\text { Thermosetting } \\
\text { Hybrid } \\
\text { Polymer }\end{array}$ & $\begin{array}{l}\text { Stainless } \\
\text { Steel } \\
\text { Type } \\
304\end{array}$ & $\begin{array}{c}\text { Triamor } \\
\text { Corrosion } \\
\text { Protection System } \\
\text { or Type } 316 \\
\text { Stainless Steel }\end{array}$ \\
\hline $\mathrm{PH}$ & 6.5 to 9.0 & 6.5 to 9.2 & 6.5 to 9.0 & 6.5 to 9.5 \\
\hline $\begin{array}{l}\text { Total Suspended } \\
\text { Solids }\end{array}$ & $25 \mathrm{ppm}$ & $25 \mathrm{ppm}$ & $25 \mathrm{ppm}$ & $25 \mathrm{ppm}$ \\
\hline $\begin{array}{l}\text { Total Dissolved } \\
\text { Solids (TDS) }\end{array}$ & $1500 \mathrm{ppm}$ & 2050ppm & 2050ppm & $2500 \mathrm{ppm}$ \\
\hline Conductivity & $2400 \mu \mathrm{s} / \mathrm{cm}$ & $3300 \mu \mathrm{s} / \mathrm{cm}$ & $3300 \mu \mathrm{s} / \mathrm{cm}$ & $400 \mu \mathrm{s} / \mathrm{cm}$ \\
\hline $\begin{array}{l}\text { Alkalinity } \\
\text { as } \mathrm{CaCO}_{3}\end{array}$ & $500 \mathrm{ppm}$ & 600ppm & 600ppm & $600 \mathrm{ppm}$ \\
\hline $\begin{array}{l}\text { Calcium Hardness } \\
\text { as } \mathrm{CaCO}_{3}\end{array}$ & $50-600 \mathrm{ppm}$ & 50-750ppm & 50-750ppm & $50-750 \mathrm{ppm}$ \\
\hline Chlorides & 250ppm & 300ppm & 300ppm & $750 \mathrm{ppm}$ \\
\hline Sulfates & 250ppm & $350 \mathrm{ppm}$ & 350ppm & 750ppm \\
\hline Silica & 150ppm & 150ppm & 150ppm & 150ppm \\
\hline
\end{tabular}

Table (2) Common Scale in Cooling Water Systems

\begin{tabular}{|c|c|}
\hline Most Common & Less Frequent \\
\hline Calcium Carbonate & Iron Oxide \\
\hline Calcium Sulphate & Zinc Phosphate \\
\hline Calcium Phosphate & Calcium Fluoride \\
\hline Magnesium Silicate & Iron Carbonate \\
\hline Silica & Aluminum Oxide \\
\hline
\end{tabular}




\section{Description of Corrosion and Scale Problems at Basrah Refinery / South Refineries Company (SRC):}

Basrah refinery (BR) is one of most refineries subsidiary South Refineries Company (SRC) located in Basrah in the south of Iraq. In Basrah refinery, water is used for the cooling towers installed in the refinery as a heat transfer medium. Feed Water supplied to the installed (BR) cooling towers is received from only single source (Alarab River). The feed water is treated in Almoftya Water Treatment Station, where the water passes by different stages of treatment before entering to the Basrah refinery. The main stages of treatment in the station are:

- Mechanical Filtration.

- Coagulation - Flocculation.

- Sand Filtration.

- Dosing of chlorine gas.

Figure (1) shows the schematic diagram of water treatment process in Almoftya Water Treatment Station. The water losses in cooling towers are replenished which is called makeup water. At present, (BR) Cooling tower efficiency enhanced by the addition of certain water treatment chemicals to increase the solubility of calcium salts, mitigate corrosion, minimize fouling and control the growth of microbiological organisms like algae, bacteria, mold and fungi, as shown in Fig. Makeup water feeds to (BR) cooling units submits to some treatments to be improved quality, but it is not enough to make the water safe to be used in cooling towers. 


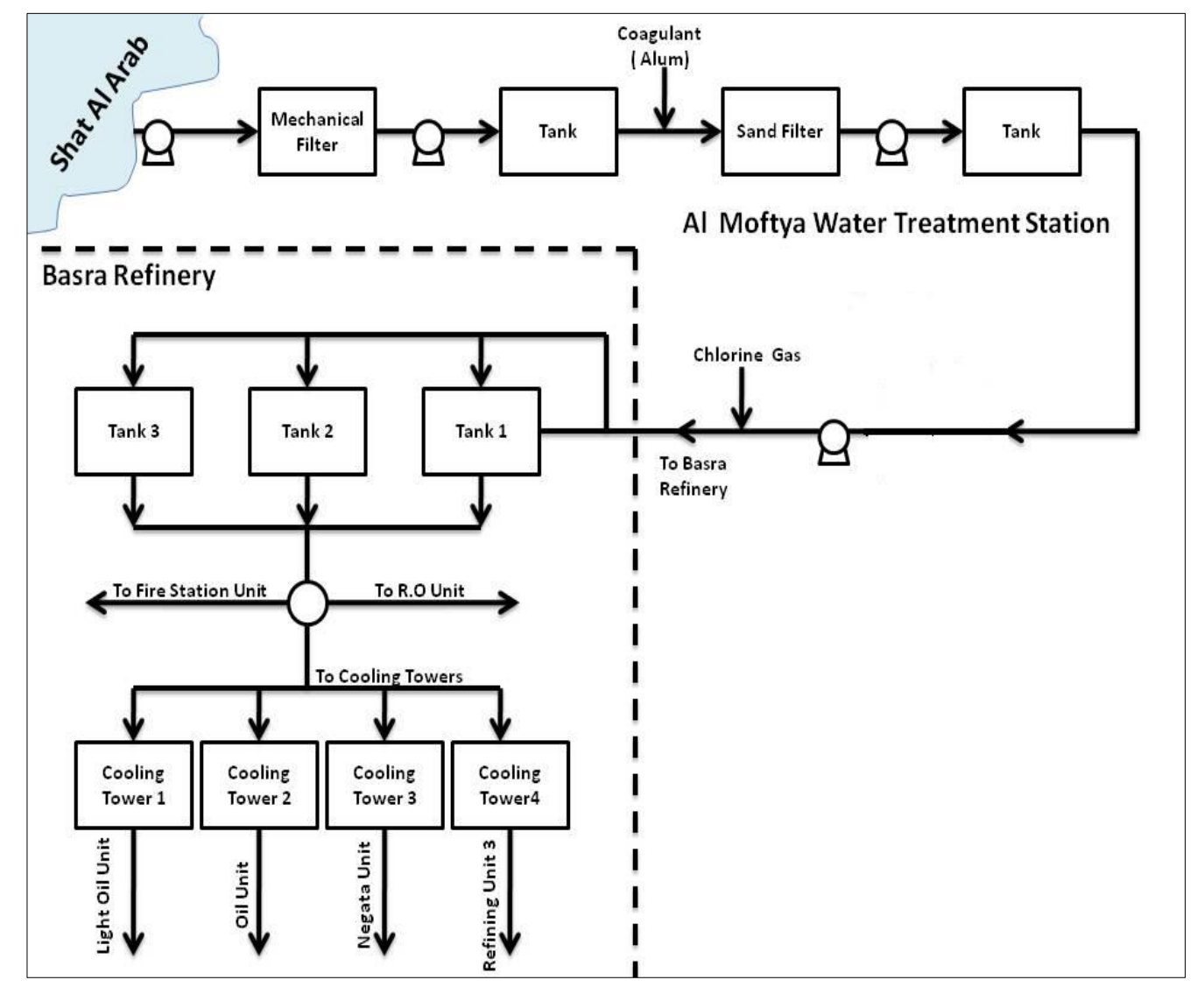

Fig. (1) Water Treatment Process in Almoftya Station

\section{Present Situation of cooling towers at SRC}

Based the Field data collected from SRC cooling towers of Basrah Refinery in different times and compared these data with the optimal required specifications of cooling towers showed, that:

- The concentrations of total dissolved salts (TDS) are higher than the acceptable limits of water used in cooling towers and that can cause scale problem.

- $\mathrm{PH}$ value of water is seemed to be with acceptable limits (6.5-8.5).

The Cooling tower efficiency of BR enhanced by the addition of certain makeup water treatment chemicals to increase the solubility of calcium salts, mitigate corrosion, minimize fouling and controls the growth of microbiological organisms like algae, bacteria, mold and fungi, as shown in Fig. However, addition of these improvers has not effect on quality of water where fouling and scales are causing 
problems in heat exchangers and pipelines, also corrosion products is found in the circulating water as well as finding of microbiological growth.

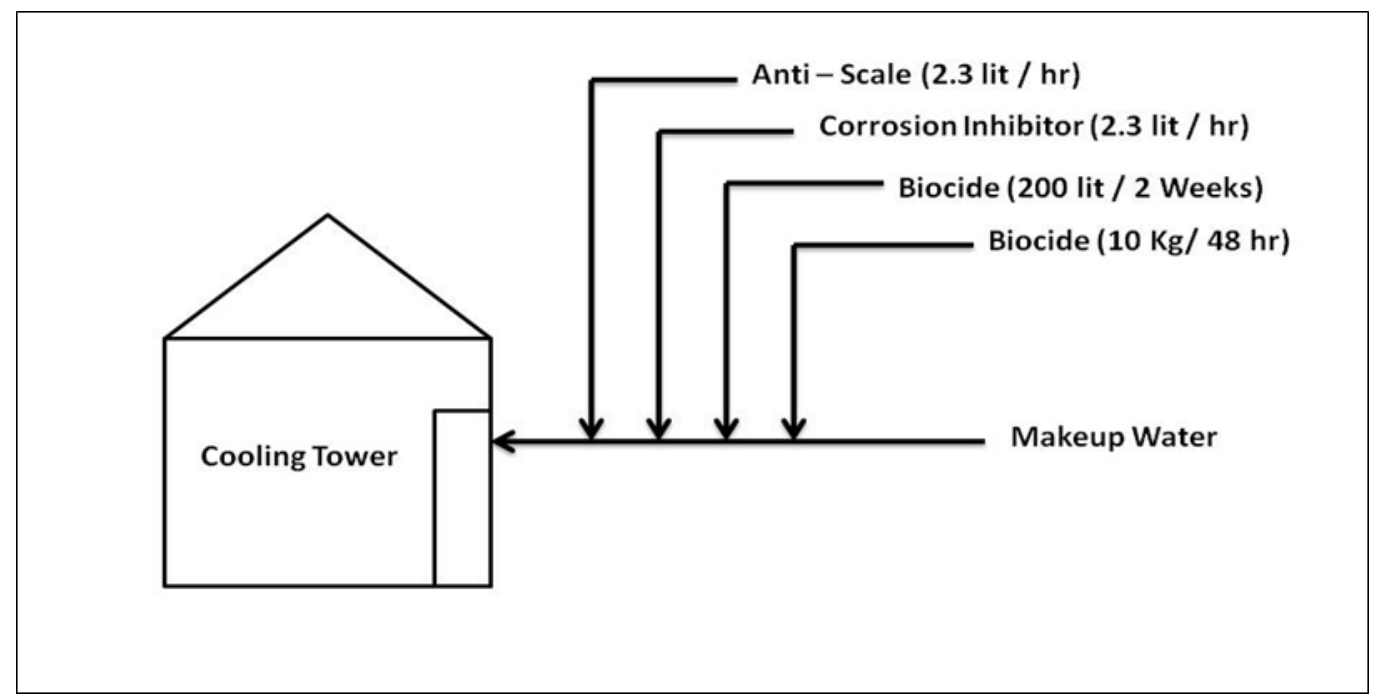

Fig. (2) Chemicals adding to makeup water at (BR)

\section{Results and Discussion}

In the current work, the field data was provided by SRC/Basrah Refinery related to the water quality that used in cooling towers. The average data of water cooling towers calculated is shown in Table (3). 
Table (3) Average calculated concentrations of cooling towers water data reported in SRC

\begin{tabular}{|c|c|c|c|c|c|c|c|c|}
\hline $\begin{array}{c}\text { Cooling* } \\
\text { Towers }\end{array}$ & $\mathbf{p H}$ & $\begin{array}{c}\text { Conduc } \\
\text { tivity } \\
\text { us. cm }\end{array}$ & \multicolumn{2}{|c|}{$\begin{array}{c}\text { Total Hardness } \\
\text { as } \mathbf{C a C O}_{\mathbf{3}} \mathbf{m g} / \mathbf{l}\end{array}$} & $\begin{array}{c}\mathbf{H C O}_{3} \\
\mathbf{m g} / \mathbf{l}\end{array}$ & $\begin{array}{c}\mathbf{S i O}_{2} \\
\mathbf{m g} / \mathbf{l}\end{array}$ & $\begin{array}{c}\text { Turbidity } \\
\text { NTU }\end{array}$ & $\begin{array}{c}\text { T.D.S } \\
\mathbf{m g} / \mathbf{l}\end{array}$ \\
\hline OLD & 7.6 & 6630 & 924 & 249 & 11.75 & 17.4 & 5206 \\
\hline NIGATTA & 8.0 & 9811 & 403 & 322.6 & 10.7 & 8.76 & 7805 \\
\hline OILS & 8.0 & 12887 & 286 & 228 & 9.0 & 8.1 & 5014 \\
\hline $\begin{array}{c}\text { IMPROVE- } \\
\text { MENT Of } \\
\text { GASOLINE/3 }\end{array}$ & 6.7 & 1917 & 50.5 & 144 & 9.4 & 7.7 & 1454 \\
\hline FEED & 6.9 & 7556 & 259 & 214 & 8.25 & 12.1 & 4892 \\
\hline
\end{tabular}

*The names of cooling towers at the first column in the table are as reported in the documents of BR and it will be written down by these names in all items of this research.

\subsection{Data Analysis by Langelier Model}

Langelier equation is the ordinary procedure using to predict occurrence of calcium carbonate scale. The $\mathrm{PH}$ value of saturation with $\mathrm{CaCO}_{3}\left(\mathrm{pH}_{\mathrm{s}}\right)$ is illustrated as bellow:

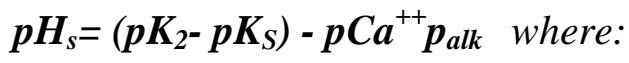

$p K_{2}=$ Constant

$p \boldsymbol{K}_{S}=$ Constant

$\boldsymbol{p} \boldsymbol{C a}^{++}=$negative logarithm of calcium concentration (ppm)

$\boldsymbol{p}_{\text {alk }}=$ negative logarithm of total alkalinity (meq/liter)

When the concentration of TDS is more than $5000 \mathrm{mg} / \mathrm{l}$, Langelier index will be affected. Stiff and Davis have simplified estimated calculation to use the equation in saline water as bellow [6]:

$p H_{s}=p C a^{++}+p_{a l k}+k$ 
$\boldsymbol{k}=$ Constant and its value depends on TDS and temperature.

The final langelier equation is:

$L S I=p H-p H_{S}$

Where:

$\boldsymbol{L S I}=$ langelier Saturation Index

$\boldsymbol{P H}=$ Real $\mathrm{PH}$ value of water (measured)

$\boldsymbol{p} \boldsymbol{H}_{S}=\mathrm{pH}$ saturation (calculated)

\subsection{Prediction of Scale and Corrosion of Water}

Applying of langelier Saturation Index on collected field data leads to predict the behavior of cooling water by comparing the results of LSI values with standard limitations, the standard limitations of LSI can be concluded as Table (4) [7]:

Table (4): Tendency of water based on LSI value

\begin{tabular}{|c|c|}
\hline LSI & Indication \\
\hline LSI $<$ Zero & $\begin{array}{c}\text { Water is under saturated with respect to calcium carbonate. Under } \\
\text { saturated water has a tendency to remove }\end{array}$ \\
\hline LSI=Zero & $\begin{array}{c}\text { Water is considered to be neutral. Neither scale forming nor scale } \\
\text { removing. }\end{array}$ \\
\hline LSI > Zero & $\begin{array}{l}\text { Water is supersaturated with respect to calcium carbonate and } \\
\text { scale forming may occur }\end{array}$ \\
\hline
\end{tabular}

Source: (langelier saturation index - Lenntech, www.lenntech.com)

\subsection{Applying of LSI on Water used in cooling towers of SRC}

Applying of LSI model on collected field cooling water data reported from SRC / Basrah Refinery Tables $(5,7,9,11,13)$, gives the following results which are obtained in Tables $(6,8,10,12,14$ respectively). 
Table (5) Water Analysis of Sample (1)

\begin{tabular}{|c|c|c|c|c|c|c|}
\hline Sample Date & \multirow{2}{*}{\multicolumn{6}{|c|}{ Water Analysis }} \\
\hline $26 / 5 / 2015$ & & & & & & \\
\hline Test & Unit & $O L D^{*}$ & $\begin{array}{c}\text { NIGAT } \\
\text { TA }\end{array}$ & OILS & FEED & $\begin{array}{c}\text { IMPROVE- } \\
\text { MENT Of } \\
\text { GASOLINE/3 }\end{array}$ \\
\hline PH & ............. & 7.8 & 8.3 & 8.4 & 8.0 & 7.7 \\
\hline Conductivity & $\mathrm{us}^{-\mathrm{cm}^{-1}}$ & 4120 & 5740 & 4950 & 3900 & 982 \\
\hline $\begin{array}{l}\text { Total Hardness as } \\
\mathrm{CaCO}_{3}\end{array}$ & $\mathrm{mg} / \mathrm{l}$ & 1048 & 1390 & 1120 & 928 & 137 \\
\hline $\mathbf{C a}^{+2}$ & $\mathrm{mg} / \mathrm{l}$ & 236 & 315 & 276 & 199 & 27 \\
\hline $\mathrm{Mg}^{+2}$ & $\mathrm{mg} / \mathrm{l}$ & 112 & 147 & 105 & 105 & 16 \\
\hline $\mathrm{Na}^{+}$ & $\mathrm{mg} / \mathrm{l}$ & 613 & 771 & 663 & 557 & 115 \\
\hline $\mathrm{SO}_{4}{ }^{-2}$ & $\mathrm{mg} / \mathrm{l}$ & 706 & 994 & 882 & 672 & 175 \\
\hline $\mathrm{HCO3}^{-}$ & $\mathrm{mg} / \mathrm{l}$ & 234 & 234 & 234 & 204 & 39 \\
\hline $\mathbf{F e}$ & $\mathrm{mg} / \mathrm{l}$ & 1.5 & 2.6 & 0.9 & 2.5 & 3.1 \\
\hline Suspended solid & $\mathrm{mg} / \mathrm{l}$ & 10.7 & 13 & 26 & 18 & 23 \\
\hline $\mathrm{SiO}_{2}$ & $\mathrm{mg} / \mathrm{l}$ & 7 & 9 & 7 & 7 & 3 \\
\hline $\begin{array}{l}\text { C.O.D }\left(\mathrm{K}_{2} \mathrm{Cr}_{2} \mathrm{O}_{7}\right. \\
\text { method) }\end{array}$ & $\mathrm{mg} / \mathrm{l}$ & 48 & 34 & 16 & 26 & Nil \\
\hline B.O.D & $\mathrm{mg} / \mathrm{l}$ & 35 & 19 & 12 & 13 & Nil \\
\hline Oil in water & $\mathrm{mg} / \mathrm{l}$ & 13.0 & Nil & Nil & --- & Nil \\
\hline Turbidity & N.T.U & 11 & 5 & 8 & 21 & 27 \\
\hline $\mathbf{K}^{+}$ & $\mathrm{mg} / \mathrm{l}$ & 10 & 15 & 15 & 10 & 30 \\
\hline T.D.S & $\mathrm{mg} / \mathrm{l}$ & 2877 & 3895 & 3218 & 2626 & 560 \\
\hline $\mathrm{PO}_{4}^{-3}$ & $\mathrm{mg} / \mathrm{l}$ & 3.8 & 8.9 & 1.36 & --- & 4.9 \\
\hline $\mathrm{O}_{2}$ & $\mathrm{mg} / \mathrm{l}$ & 2.4 & 8.0 & 6.6 & 6.7 & 7.5 \\
\hline AcidophIllic & $\begin{array}{c}\text { colony/ } \\
\mathrm{ml}\end{array}$ & $10^{5}$ & $10^{5}$ & $10^{3}$ & $10^{4}$ & $10^{4}$ \\
\hline T.anerobic & $\begin{array}{c}\text { colony/ } \\
\mathrm{ml}\end{array}$ & $10^{5}$ & $10^{5}$ & $10^{4}$ & $10^{4}$ & $10^{5}$ \\
\hline S.R.B & $\begin{array}{c}\text { colony/ } \\
\mathrm{ml}\end{array}$ & $10^{3}$ & $10^{5}$ & $10^{2}$ & $10^{2}$ & $10^{2}$ \\
\hline
\end{tabular}

Table (6) Applying of LSI Model on Sample (1) Data

\begin{tabular}{|c|c|c|c|}
\hline C.W & PHs & LSI & Description \\
\hline OLD & 6.7 & $\mathbf{1 . 1}$ & scale forming but non corrosive \\
\hline NIGATTA & 6.7 & $\mathbf{1 . 6}$ & scale forming but non corrosive \\
\hline OILS & 6.7 & $\mathbf{1 . 7}$ & scale forming but non corrosive \\
\hline FEED & 6.8 & $\mathbf{1 . 2}$ & scale forming but non corrosive \\
\hline $\begin{array}{c}\text { IMPROVE-MENT } \\
\text { Of GASOLINE }\end{array}$ & 8.1 & $\mathbf{- 0 . 4 5}$ & slightly corrosive but non -scale forming \\
\hline
\end{tabular}


Table (7) Water Analysis of Sample (2)

\begin{tabular}{|c|c|c|c|c|}
\hline $\begin{array}{c}\text { Sample Date } \\
9 / 6 / 2015 \\
\end{array}$ & & & & \\
\hline Test & Unit & OLD & NIGATTA & FEED \\
\hline PH & $\ldots \ldots+\cdots$ & 7.8 & 8.2 & 7.9 \\
\hline Conductivity & us-cm $^{-1}$ & 4300 & 6190 & 3720 \\
\hline $\begin{array}{l}\text { Total Hardness as } \\
\mathrm{CaCO}_{3}\end{array}$ & $\mathrm{mg} / \mathrm{l}$ & 1058 & 1508 & 890 \\
\hline $\mathrm{Ca}^{+2}$ & $\mathrm{mg} / \mathrm{l}$ & 242 & 322 & 199 \\
\hline $\mathrm{Mg}^{+2}$ & $\mathrm{mg} / \mathrm{l}$ & 111 & 171 & 96 \\
\hline $\mathrm{Na}^{+}$ & $\mathrm{mg} / \mathrm{l}$ & 615 & 1032 & 435 \\
\hline $\mathrm{SO}_{4}{ }^{-2}$ & $\mathrm{mg} / \mathrm{l}$ & 630 & 900 & 592 \\
\hline $\mathrm{Cl}^{-}$ & $\mathrm{mg} / \mathrm{l}$ & 962 & 1612 & 861 \\
\hline $\mathrm{HCO3}^{-}$ & $\mathrm{mg} / \mathrm{l}$ & 224 & 331 & 195 \\
\hline $\mathbf{F e}$ & $\mathrm{mg} / \mathrm{l}$ & 2.2 & 0.95 & 0.96 \\
\hline Suspended solid & $\mathrm{mg} / \mathrm{l}$ & 39 & 9.0 & 4.0 \\
\hline $\mathrm{SiO}_{2}$ & $\mathrm{mg} / \mathrm{l}$ & 12 & 13 & 10 \\
\hline $\begin{array}{l}\text { C.O.D }\left(\mathrm{K}_{2} \mathrm{Cr}_{2} \mathrm{O}_{7}\right. \\
\text { method) }\end{array}$ & $\mathrm{mg} / \mathrm{l}$ & 75 & 420 & 15 \\
\hline B.O.D 5 & $\mathrm{mg} / \mathrm{l}$ & 56.5 & 26.6 & 14.1 \\
\hline Oil in water & $\mathrm{mg} / \mathrm{l}$ & 37 & 34 & - \\
\hline Turbidity & N.T.U & 19 & 7.4 & 6.9 \\
\hline $\mathbf{K}^{+}$ & $\mathrm{mg} / \mathrm{l}$ & 15 & 15 & 10 \\
\hline T.D.S & $\mathrm{mg} / \mathrm{l}$ & 2813 & 4396 & 2399 \\
\hline $\mathrm{PO}_{4}{ }^{-3}$ & $\mathrm{mg} / \mathrm{l}$ & 5.6 & 1.7 & 1.18 \\
\hline $\mathbf{O}_{2}$ & $\mathrm{mg} / \mathrm{l}$ & 2.6 & 2.2 & 5.3 \\
\hline AcidophIllic & colony/ml & $10^{3}$ & $10^{2}$ & $10^{2}$ \\
\hline T.anerobic & colony/ml & $10^{5}$ & $10^{5}$ & $10^{4}$ \\
\hline S.R.B & colony/ml & $10^{2}$ & $10^{2}$ & $10^{2}$ \\
\hline
\end{tabular}

Table (8) Applying of LSI Model on Sample (2) Data

\begin{tabular}{|c|c|c|c|}
\hline C.W & PHs & LSI & Description \\
\hline$O L D$ & 6.7 & 1.1 & scale forming but non corrosive \\
\hline NIGATTA & 6.5 & 1.7 & scale forming but non corrosive \\
\hline OILS & ------ & ----- & - \\
\hline FEED & 6.8 & 1.1 & scale forming but non corrosive \\
\hline $\begin{array}{c}\text { IMPROVE-MENT } \\
\text { Of GASOLINE }\end{array}$ & ------ & ----- & \\
\hline
\end{tabular}


Table (9) Water Analysis of Sample (3)

\begin{tabular}{|c|c|c|c|c|c|c|}
\hline Sample Date & \multicolumn{6}{|c|}{ Water Analysis } \\
\hline Test & Unit & $O L D$ & $\begin{array}{c}\text { NIGATT } \\
A\end{array}$ & $\begin{array}{c}\text { OIL } \\
S\end{array}$ & FEED & $\begin{array}{c}\text { IMPROVE- } \\
\text { MENT Of } \\
\text { GASOLINE /3 }\end{array}$ \\
\hline PH & …......... & 7.6 & 7.9 & & 7.8 & 7.9 \\
\hline Conductivity & ${\text { us- }-\mathrm{cm}^{-1}}^{-1}$ & 11280 & 15100 & & 10500 & 3860 \\
\hline $\begin{array}{l}\text { Total Hardness } \\
\text { as } \mathrm{CaCO}_{3}\end{array}$ & $\mathrm{mg} / \mathrm{l}$ & 1922 & 2516 & & 1804 & 360 \\
\hline $\mathrm{Ca}^{+2}$ & $\mathrm{mg} / \mathrm{l}$ & 348 & 450 & & 312 & 66 \\
\hline $\mathrm{Mg}^{+2}$ & $\mathrm{mg} / \mathrm{l}$ & 256 & 339 & & 249 & 47 \\
\hline $\mathrm{Na}^{+}$ & $\mathrm{mg} / \mathrm{l}$ & 2398 & 3185 & & 2129 & 522 \\
\hline $\mathrm{SO}_{4}^{-2}$ & $\mathrm{mg} / \mathrm{l}$ & 932 & 1428 & & 1048 & 812 \\
\hline $\mathrm{Cl}^{-}$ & $\mathrm{mg} / \mathrm{l}$ & 3748 & 4978 & & 3328 & 816 \\
\hline $\mathrm{HCO3}^{-}$ & $\mathrm{mg} / \mathrm{l}$ & 244 & 280 & & 195 & 102 \\
\hline $\mathbf{F e}$ & $\mathrm{mg} / \mathrm{l}$ & 1.03 & 2.2 & & 0.72 & 3.6 \\
\hline Suspended solid & $\mathrm{mg} / \mathrm{l}$ & 19 & 21 & & 7.0 & 3.0 \\
\hline $\mathrm{SiO}_{2}$ & $\mathrm{mg} / \mathrm{l}$ & 12 & 10 & & 9 & 6 \\
\hline $\begin{array}{l}\text { C.O.D } \\
\left(\mathrm{K}_{2} \mathrm{Cr}_{2} \mathrm{O}_{7} \text { method }\right)\end{array}$ & $\mathrm{mg} / \mathrm{l}$ & 91 & 122 & & 41 & 75 \\
\hline B.O.D & $\mathrm{mg} / \mathrm{l}$ & 32 & 43 & & 18 & 15 \\
\hline Oil in water & $\mathrm{mg} / \mathrm{l}$ & 8.5 & 21 & & - & NIL \\
\hline Turbidity & N.T.U & 6.0 & 6.5 & & 3.8 & 4.5 \\
\hline $\mathbf{K}^{+}$ & $\mathrm{mg} / \mathrm{l}$ & 20 & 30 & & 20 & 5 \\
\hline T.D.S & $\mathrm{mg} / \mathrm{l}$ & 7958 & 10700 & & 7290 & 2379 \\
\hline $\mathrm{PO}_{4}^{-3}$ & $\mathrm{mg} / \mathrm{l}$ & 5.1 & 10.2 & & - & 3.5 \\
\hline $\mathbf{O}_{2}$ & $\mathrm{mg} / \mathrm{l}$ & 1.8 & 2.9 & & 5.3 & 7.4 \\
\hline AcidophIllic & colony/ml & & & & & \\
\hline T.anerobic & colony/ml & & & & & \\
\hline S.R.B & colony/ml & & & & & \\
\hline
\end{tabular}

Table (10) Applying of LSI Model on Sample (3) Data

\begin{tabular}{|c|c|c|c|}
\hline C.W & PHs & LSI & Description \\
\hline OLD & 6.8 & 1.0 & Scale forming but non corrosive \\
\hline NIGATTA & 6.7 & 1.2 & Scale forming but non corrosive \\
\hline OILS & ---- & ---- & -------------------------------------------- \\
\hline FEED & 6.9 & 0.91 & Scale forming but non corrosive \\
\hline $\begin{array}{l}\text { IMPROVE-MENT } \\
\text { Of GASOLINE/3 }\end{array}$ & 7.6 & 0.30 & Slightly scale forming and corrosive \\
\hline
\end{tabular}


Table (11) Water Analysis of Sample (4)

\begin{tabular}{|c|c|c|c|c|c|}
\hline $\begin{array}{c}\text { Sample Date } \\
17 / 11 / 2015 \\
\end{array}$ & & & & & \\
\hline Test & Unit & OLD & $\begin{array}{r}\text { OIL } \\
\mathbf{S}\end{array}$ & Feed & $\begin{array}{l}\text { IMPROVE-MENT } \\
\text { Of GASOLINE /3 }\end{array}$ \\
\hline PH & 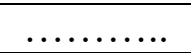 & 7.4 & 7.7 & 7.1 & 7.2 \\
\hline Conductivity & us-cm $^{-1}$ & 9610 & 7700 & 9670 & 1990 \\
\hline Total Hardness as $\mathrm{CaCO}_{3}$ & $\mathrm{mg} / \mathrm{l}$ & 1572 & 1244 & 1522 & 240 \\
\hline $\mathrm{Ca}^{+2}$ & $\mathrm{mg} / \mathrm{l}$ & 317 & 252 & 308 & 52 \\
\hline $\mathrm{Mg}^{+2}$ & $\mathrm{mg} / \mathrm{l}$ & 190 & 150 & 183 & 27 \\
\hline $\mathrm{Na}^{+}$ & $\mathrm{mg} / \mathrm{l}$ & 1750 & 1301 & 1722 & 316 \\
\hline $\mathrm{SO}_{4}^{-2}$ & $\mathrm{mg} / \mathrm{l}$ & 1000 & 908 & 1204 & 170 \\
\hline $\mathrm{Cl}^{-}$ & $\mathrm{mg} / \mathrm{l}$ & 2734 & 2033 & 2692 & 495 \\
\hline $\mathrm{HCO3}^{-}$ & $\mathrm{mg} / \mathrm{l}$ & 244 & 195 & 240 & 136 \\
\hline $\mathbf{F e}$ & $\mathrm{mg} / \mathrm{l}$ & 0.82 & 0.7 & 1.3 & 0.7 \\
\hline Suspended solid & $\mathrm{mg} / \mathrm{l}$ & 25 & 17 & 12 & 2 \\
\hline $\mathrm{SiO}_{2}$ & $\mathrm{mg} / \mathrm{l}$ & 13 & 10 & 12 & 8 \\
\hline C.O.D $\left(\mathrm{K}_{2} \mathrm{Cr}_{2} \mathrm{O}_{7}\right.$ method $)$ & $\mathrm{mg} / \mathrm{l}$ & 95 & 31.9 & 31.6 & 61 \\
\hline B.O.D5 & $\mathrm{mg} / \mathrm{l}$ & 56 & 21.5 & 7.9 & 32.8 \\
\hline Oil in water & $\mathrm{mg} / \mathrm{l}$ & 29 & 2.4 & - & 32 \\
\hline Turbidity & N.T.U & 7.7 & 3.6 & 16.8 & 5.2 \\
\hline $\mathbf{K}^{+}$ & $\mathrm{mg} / \mathrm{l}$ & 15 & 10 & 15 & 5 \\
\hline T.D.S & $\mathrm{mg} / \mathrm{l}$ & 0.9 & 4859 & 6376 & 7.5 \\
\hline $\mathrm{PO}_{4}^{-3}$ & $\mathrm{mg} / \mathrm{l}$ & 0.4 & 0.2 & - & 0.2 \\
\hline $\mathbf{O}_{2}$ & $\mathrm{mg} / \mathrm{l}$ & 7.0 & 7.8 & 6.0 & 0.6 \\
\hline AcidophIllic & colony/ml & 6263 & $10^{2}$ & $10^{5}$ & $10^{5}$ \\
\hline T.anerobic & colony/ml & $10^{5}$ & $10^{2}$ & $10^{5}$ & $10^{5}$ \\
\hline S.R.B & colony/ml & $10^{5}$ & $10^{3}$ & $10^{3}$ & $10^{5}$ \\
\hline
\end{tabular}

Table (12) Applying of LSI Model on Sample (4) Data

\begin{tabular}{|c|c|c|c|}
\hline C.W & PHs & LSI & Description \\
\hline OLD & 6.8 & $\mathbf{0 . 6 3}$ & Scale forming but non corrosive \\
\hline NIGATTA & ---- & ---- & ----------- \\
\hline OILS & 6.9 & $\mathbf{0 . 8 0}$ & Scale forming but non corrosive \\
\hline FEED & 6.8 & $\mathbf{0 . 3 1}$ & Slightly scale forming and corrosive \\
\hline $\begin{array}{c}\text { IMPROVE-MENT } \\
\text { Of GASOLINE/3 }\end{array}$ & 7.4 & $\mathbf{- 0 . 2 4}$ & Slightly corrosive but non scale forming \\
\hline
\end{tabular}


Table (13) Water Analysis of Sample (5)

\begin{tabular}{|c|c|c|c|c|c|}
\hline $\begin{array}{c}\text { Sample Date } \\
3 / 11 / 2015 \\
\end{array}$ & & & & & \\
\hline Test & Unit & $O L D$ & NIGATAT & OILS & FEED \\
\hline $\mathbf{P H}$ & ......... & 7.5 & 7.8 & 7.8 & 7.7 \\
\hline Conductivity & ${\mathrm{us}-\mathrm{cm}^{-1}}^{-1}$ & 4430 & 6860 & 5760 & 4520 \\
\hline $\begin{array}{c}\text { Total Hardness as } \\
\mathrm{CaCO}_{3}\end{array}$ & $\mathrm{mg} / \mathrm{l}$ & 920 & 1400 & 1120 & 900 \\
\hline $\mathrm{Ca}^{+2}$ & $\mathrm{mg} / \mathrm{l}$ & 196 & 304 & 208 & 180 \\
\hline $\mathrm{Mg}^{+2}$ & $\mathrm{mg} / \mathrm{l}$ & 105 & 156 & 146 & 109 \\
\hline $\mathrm{Na}^{+}$ & $\mathrm{mg} / \mathrm{l}$ & 711 & 1154 & 942 & 739 \\
\hline $\mathrm{SO}_{4}{ }^{-2}$ & $\mathrm{mg} / \mathrm{l}$ & 435 & 924 & 752 & 712 \\
\hline $\mathrm{Cl}^{-}$ & $\mathrm{mg} / \mathrm{l}$ & 1111 & 1803 & 1473 & 1155 \\
\hline $\mathrm{HCO3}^{-}$ & $\mathrm{mg} / \mathrm{l}$ & 239 & 213 & 198 & 195 \\
\hline $\mathrm{Fe}$ & $\mathrm{mg} / \mathrm{l}$ & 1.6 & 1.7 & 1.4 & 1.0 \\
\hline Suspended solid & $\mathrm{mg} / \mathrm{l}$ & 8 & 12 & 8 & 5 \\
\hline $\mathrm{SiO}_{2}$ & $\mathrm{mg} / \mathrm{l}$ & 11 & 13 & 10 & 8 \\
\hline $\begin{array}{c}\text { C.O.D }\left(\mathrm{K}_{2} \mathrm{Cr}_{2} \mathrm{O}_{7}\right. \\
\text { method) }\end{array}$ & $\mathrm{mg} / \mathrm{l}$ & 68 & 26 & 21 & 21 \\
\hline B.O.D 5 & $\mathrm{mg} / \mathrm{l}$ & 24 & 12 & 9 & 5.7 \\
\hline Oil in water & $\mathrm{mg} / \mathrm{l}$ & 35 & 17 & 14 & - \\
\hline Turbidity & N.T.U & 19 & 20 & 14 & 8.0 \\
\hline $\mathbf{K}^{+}$ & $\mathrm{mg} / \mathrm{l}$ & 10 & 15 & 10 & 10 \\
\hline T.D.S & $\mathrm{mg} / \mathrm{l}$ & 2820 & 4582 & 3740 & 3109 \\
\hline $\mathrm{PO}_{4}{ }^{-3}$ & $\mathrm{mg} / \mathrm{l}$ & 2.3 & 7.6 & 6.8 & 7.2 \\
\hline $\mathbf{O}_{2}$ & $\mathrm{mg} / \mathrm{l}$ & 3.7 & 5.4 & 1.5 & - \\
\hline AcidophIllic & colony/ml & 0.4 & 0.3 & 0.3 & 0.2 \\
\hline T.anerobic & colony/ml & $10^{3}$ & $10^{4}$ & $10^{4}$ & $10^{4}$ \\
\hline S.R.B & colony/ml & $10^{5}$ & $10^{5}$ & $10^{5}$ & \\
\hline
\end{tabular}

Table (14) Applying of LSI Model on Sample (5) Data

\begin{tabular}{|c|c|c|l|}
\hline C.W & PHs & LSI & Description \\
\hline OLD & $\mathbf{6 . 7}$ & $\mathbf{0 . 8 0}$ & Scale forming but non corrosive \\
\hline NIGATTA & $\mathbf{6 . 7}$ & $\mathbf{1 . 1}$ & Scale forming but non corrosive \\
\hline OILS & $\mathbf{6 . 8}$ & $\mathbf{0 . 9 8}$ & Scale forming but non corrosive \\
\hline FEED & $\mathbf{6 . 8}$ & $\mathbf{0 . 8 7}$ & Scale forming but non corrosive \\
\hline $\begin{array}{c}\text { IMPROVE-MENT Of } \\
\text { GASOLINE }\end{array}$ & ----- & & ----------------------------- \\
\hline
\end{tabular}




\section{Conclusions}

The obtained results collected by applying LSI model on cooling water analysis data show that the tendency of water to form scale $\left(\mathrm{CaCO}_{3}\right.$ Precipitation) at units (OLD, NIGATTA, OILS). Also, the feed water has the same tendency for scale forming. Applying of LSI model on data analysis of cooling water for (IMPROVEMENT of GASOLINE) shows the tendency of water to be slightly corrosive without forming of scale. Bad quality of water parameters is noticed from high levels of calcium and alkalinity, which leads to high value of PHs (PH at saturation), causes scale forming and corrosion. 


\section{$\underline{\text { References }}$}

[1] Cooling Tower: Heat Transfer Equipment in Process Industry,http://www.piping-enginnering.

[2] Washington State Air Toxic Sources and Emission Estimation Methods), $\underline{\text { sortress.wa.gov }>\text { ecy }>\text { publications }>\text { documents }}$

[3] Code of Practice for Fresh Water Cooling Towers, Part 1: Design, Installation and Commissioning, www.emsd.gov.hk>filemanager>content_296>fwctcop_part_1

[4] Corrosion and Scale Control ;Biological Control -Bac 3240C www.manualslib.com>... $>3240 \mathrm{C}>$ operation 2 maintenance manual

[5] Water Quality guidelines, www.baltimoreaircoil.com/English/resourcelibrary/file/579.

[6] Corrosion and Water Technology in petroleum and Gas Fields, Mohammed Ahmed Khalil, 2006 (The reference is written in Arabic).

[7] https://www.lenntech.com/calculators/langelier/index/langelier.htm 